\title{
Lanthanoid Staining as a Fast Technology of Preparing Microbiological Specimens for Scanning Electron Microscopy
}

DOI: $10.17691 / \mathrm{stm} 2017.9 .3 .03$

Received May 11, 2017

I.V. Chebotar, MD, DSc, Leading Researcher, Microbiology Laboratory'; Leading Researcher,

Laboratory of Microbiology and Antibacterial Therapy²;

I.A. Novikov, Senior Researcher, Laboratory of Fundamental Research, Head of the Electron Microscopy Group 3 ;

A.M. Subbot, MD, PhD, Senior Researcher, Laboratory of Fundamental Research, Head of the Cellular Technologies Group ${ }^{3}$;

N.A. Mayansky, MD, DSc, Head of the Laboratory Unit ${ }^{1}$

\begin{abstract}
${ }^{1}$ National Scientific and Practical Center of Children's Health, 2/1 Lomonosovsky Avenue, Moscow, 119991, Russian Federation;

${ }^{2}$ Clinical and Research Institute of Emergency Children's Surgery and Trauma, 22 Bol'shaya Polyanka St., Moscow, 119180, Russian Federation;

${ }^{3}$ Research Institute of Eye Diseases, 11 Rossolimo St., Moscow, 119021, Russian Federation
\end{abstract}

The aim of the investigation was to demonstrate advantages of lanthanoid staining as a novel super-fast technology of preparing microbiological specimens for scanning electron microscopy, and to ground the potentials of using this technology in practical microbiology.

Materials and Methods. Microbial cells (bacteria Acinetobacter baumannii, Corynebacterium diphtheriae, Klebsiella pneumoniae, Pseudomonas aeruginosa, Staphylococcus aureus, Streptococcus pneumoniae, and fungi Candida albicans), and Staphylococcus aureusbased bacterial biofilms were stained with the preparation based on neodymium chloride and visualized using scanning electron microscopy. Observations were carried out in the mode of low vacuum, images were captured in the mode of detecting back-scattered electrons using a short working section of 4.5-3.0 mm. Specimens were compared to the untreated microorganisms or microbial samples obtained by means of traditional specimen preparation with dehydration and sputtering a gold-platinum coating.

Results. The lanthanoid staining technology enabled visualization and evaluation of diagnostically relevant morphological structures of the following microbial cells: A. baumannii, C. diphtheriae, K. pneumoniae, P. aeruginosa, S. aureus, S. pneumoniae, C. albicans, including their relative position, shape, dimensions, presence of capsules and flagella, intracellular structures (polyphosphate inclusions, septum) as well as an extracellular bacterial matrix. The obtained images were characterized by a high contrast, which may be explained by the fact that lanthanoids are capable of providing a high density of electron back-scattering under the impact of the microscope electron beam, resulting in a great brightness of the biological object loci saturated with these elements.

Conclusion. Lanthanoid staining of microorganisms is a perspective method of specimen preparation for scanning electron microscopy, which opens the possibility of practical application of this method in diagnostic technologies of clinical microbiology.

Key words: microbial cells; electron microscopy; scanning electron microscopy; microbiological specimen preparation; lanthanoids; microbiological diagnosis.

Visualization of microorganisms and their subcellular structures is of great importance for both clinical microbiology and a research work. Diverse variants of microscopy are used in microbiological practice. Some of them are routine and based on light microscopy of primitively stained specimens (for example, detection of Neisseria stained with methylene blue) or native specimens (for example, detection of Treponema by dark-field microscopy). Others are used for solving fundamental scientific tasks in sophisticated research stations, and require a complicated material preparation.
Electron microscopy has been referred until now to the second category of methods. The main obstacle to its implementation into everyday practice in microbiology is a labor-consuming, long-term and expensive preparation of biological specimens. Even the most advanced variants of specimen preparation including fixation, dehydration, preparation of ultra-thin sections (for transmission electron microscopy) or metallization (for scanning electron microscopy, SEM) take not less than $24 \mathrm{~h}$. Such terms and labor intensity make this method difficult for using in routine practice of clinical microbiology.

For contacts: Igor V. Chebotar, e-mail: nizarnn@yandex.ru 
As far as 5 years ago we were sure that SEM had no future for solving the tasks of clinical microbiology [1]. Now, multiple efforts to modify specimen preparation for electron microscopy aimed at its simplification have been reported. Priester and his colleagues [2], in particular, reduced the time of microbial biofilm preparation for SEM to $5.5 \mathrm{~h}$ owing to their original method of staining and fixation. However, even this period is too long, and fixation implies application of toxic substances (osmium tetroxide, glutaraldehyde). Another variant of a more rapid specimen preparation for SEM was realized by treating the specimen surface with concentrated acids: hydrochloric $(\mathrm{HCl})$ and hydrofluoric (HF) acids [3]. Such treatment, to the authors' opinion, had to fully replace metallization of the tested material surface. The results of approbation of this method appeared to be ambiguous due to corrosive attack of the acids on the specimen and parts of the electron microscope. In any case, the described methods failed to implement SEM into everyday microbiological practice. Meanwhile, there are data about successful use of lanthanoids (neodymium chloride) for express-preparation of some types of biological specimens for various kinds of electron microscopy $[4,5]$.

The aim of the investigation was to demonstrate advantages of lanthanoid staining as a novel superfast technology of preparing microbiological specimens for scanning electron microscopy, and to ground the potentials of using this technology in practical microbiology.

Materials and Methods. Reference strains of microorganisms received from American Type Culture Collection (Acinetobacter baumannii ATCC 19606, Pseudomonas aeruginosa ATCC 27853, Staphylococcus aureus ATCC 29213, Streptococcus pneumoniae ATCC 49619), and collection strains (Candida albicans 66-1556, Corynebacterium diphtheriae, biovar mitis, nontoxigenic strain 11-611, Klebsiella pneumoniae 36-2246) from the laboratory of microbiology of the National Scientific and Practical Center of Children's Health of the Russian Ministry of Health were used in the work.

Microbial specimens for SEM were prepared from the colonies grown on the dense culture medium, and from biofilms formed in the nutrient broth. To obtain bacteria of the colonial origin a blood agar with the addition of $3 \%$ horse serum was used. Fungi $C$. albicans were cultured on Sabouraud agar (Becton, Dickinson and Company, USA). All microorganisms, except S. pneumoniae, were cultured at $37^{\circ} \mathrm{C}$ in the air atmosphere. S. pneumoniae was cultured at $37^{\circ} \mathrm{C}$ in the presence of $5 \% \mathrm{CO}_{2}$. Cells from the colonies of 24-hours' cultures were suspended in Hanks solution without phenol red at concentration corresponding to 0.1 McFarland Standard. $0.1 \mathrm{ml}$ of suspension was applied on the polystyrene disks (7 $\mathrm{mm}$ in diameter), incubated for $15 \mathrm{~min}$ in the moist chamber at $37^{\circ} \mathrm{C}$ to provide adhesion of the cells to the surface. Immediately after that the residues of microbial suspension were removed from the disk surfaces with a micropipette, and the surface with adhered microbe cells was treated with the reagents from the BioREE kit for staining (Glaucon, Russia) according to the manufacturer's protocol. The specimens were not subjected to fixation and dehydration.

Native (unstained) microorganisms adhered to the polystyrene disk surface were used for comparison. For objectivization of the results of comparing the images of staphylococci stained with neodymium chloride and native staphylococci, histograms of brightness distribution on the scans obtained using identical capture parameters were constructed, and the difference between the two modes of brightness distribution was calculated.

In another series of the experiments, SEM images of the biofilms stained with lanthanides were compared with the biofilms prepared according to the standard method of specimen preparation for SEM. The biofilms were formed on the surface of the polystyrene disks $(7 \mathrm{~mm}$ in diameter) placed in the wells of 24-well plate (Greiner Bio-One $\mathrm{GmbH}$, Austria). A known biofilm-forming strain of staphylococcus, S. aureus ATCC 29213, was used for this purpose. Its 24 hours' culture $(0.05 \mathrm{ml}$ in volume) in Mueller-Hinton broth adjusted to 0.5 McFarland Standard was inoculated in the plate wells with $0.95 \mathrm{ml}$ of tryptic soy broth (Becton, Dickinson and Company, USA) containing $1 \%$ glucose. It was then incubated for $48 \mathrm{~h}$ at $37^{\circ} \mathrm{C}$. The disk with the biofilm was removed from the plate and rinsed twice with isotonic solution of sodium chloride. Immediately after that, the surface with the biofilm was treated with the reagents from the BioREE kit for staining (as described above). No fixation and dehydration of the specimens were done. Specimens for comparison were biofilms undergone standard specimen preparation for SEM, procedures of fixation with $2.5 \%$ glutaraldehyde, postfixation with osmium tetroxide with subsequent dehydration (graded alcohols from 20 to $97 \%$, supercritical drying), and deposition of the goldplatinum coating [6].

The unstained specimens and specimens after lanthanoid staining were secured on the microscope stage using adhesive carbon tape (Nisshin EM, Co., Japan) and placed into the chamber of the scanning electron microscope (EVO LS10; Carl Zeiss, Germany). Specimens were viewed in low vacuum at accelerating voltage of 21-27 kV and 360-520 pA current on the specimen. $\mathrm{LaB}_{6}$ cathode was used. Images were captured in the mode of back-scattered electron (BSE) detection. The picture area size was $3,072 \times 2,304$ pixels. In order to obtain a satisfactory microscope resolution in the BSE mode, viewing was performed using a 4.5$3.0 \mathrm{~mm}$ short working segment.

Specimens prepared according to the classical scheme with the sputtered gold-platinum film were secured on the microscope stage using adhesive carbon tape (Nisshin EM, Co., Japan). A conductive paste was used to drain the charge from the sputtered specimen 
surface. Viewing was done in the mode of high vacuum at accelerating voltage of $20 \mathrm{kV}$ and $260 \mathrm{kA}$ current on the specimen. $\mathrm{LaB}_{6}$ cathode was used. Images of specimen microtopography were captured in the mode of secondary electron detection.

\section{Results}

At the first stage of the work, obtained SEM images of native (unstained) microbe cell specimens were compared with the similar cells treated with lanthanoids (neodymium chloride). These images allow visual representation of differences between the native cells of S. aureus ATCC 29213 and analogous staphylococci after staining. It is clearly seen that lanthanoid staining (Figure 1 (a)) provides highly contrast images, whereas specimens of unstained staphylococci (Figure 1 (c)) are noted to have low contrast.

In case of using BSE detector, the contrast of the image being formed is determined by mean atomic mass at every point of the visualized specimen. Saturation of the specimen with the elements of Lanthanum group resulted in the substantial increase of the image contrast. Objectively, such differences in the contrast may be illustrated by histograms of brightness distribution of the compared images. In both cases, brightness of the points has a marked bimodal character (Figure 1 (b), (d)). The first mode (a set of darker points) characterizes the intensity of the electron back-scattering in the nearsurface polystyrene layer, while the second mode shows the intensity in the structures of microorganisms. Under equal condition of image capturing, the brightness of polystyrene was 64-66 standard units (SU) of digital brightness, for unstained microorganisms brightness was equal to $85 \mathrm{SU}$, and for lanthanoid stained ones it was $146 \mathrm{SU}$. In percentage terms, the contrast of the microorganisms treated with neodymium chloride relative to polystyrene substrate grew from 31.4 to $125.5 \%$. An important drawback of the image of the unstained staphylococci acquired by SEM is absence of visualization of extracellular structures: mucus, biofilm matrix.

The second stage allowed the assessment of the quality of biofilm SEM images after lanthanoid staining and standard specimen preparation including specimen dehydration and metallization of its surface (Figure 2). Lanthanoid staining of S. aureus ATCC 29213 biofilms (Figure 2 (a)) enabled detection of two main structural biofilm elements: cells and extracellular (biofilm) matrix. The cells stained with neodymium chloride demonstrated
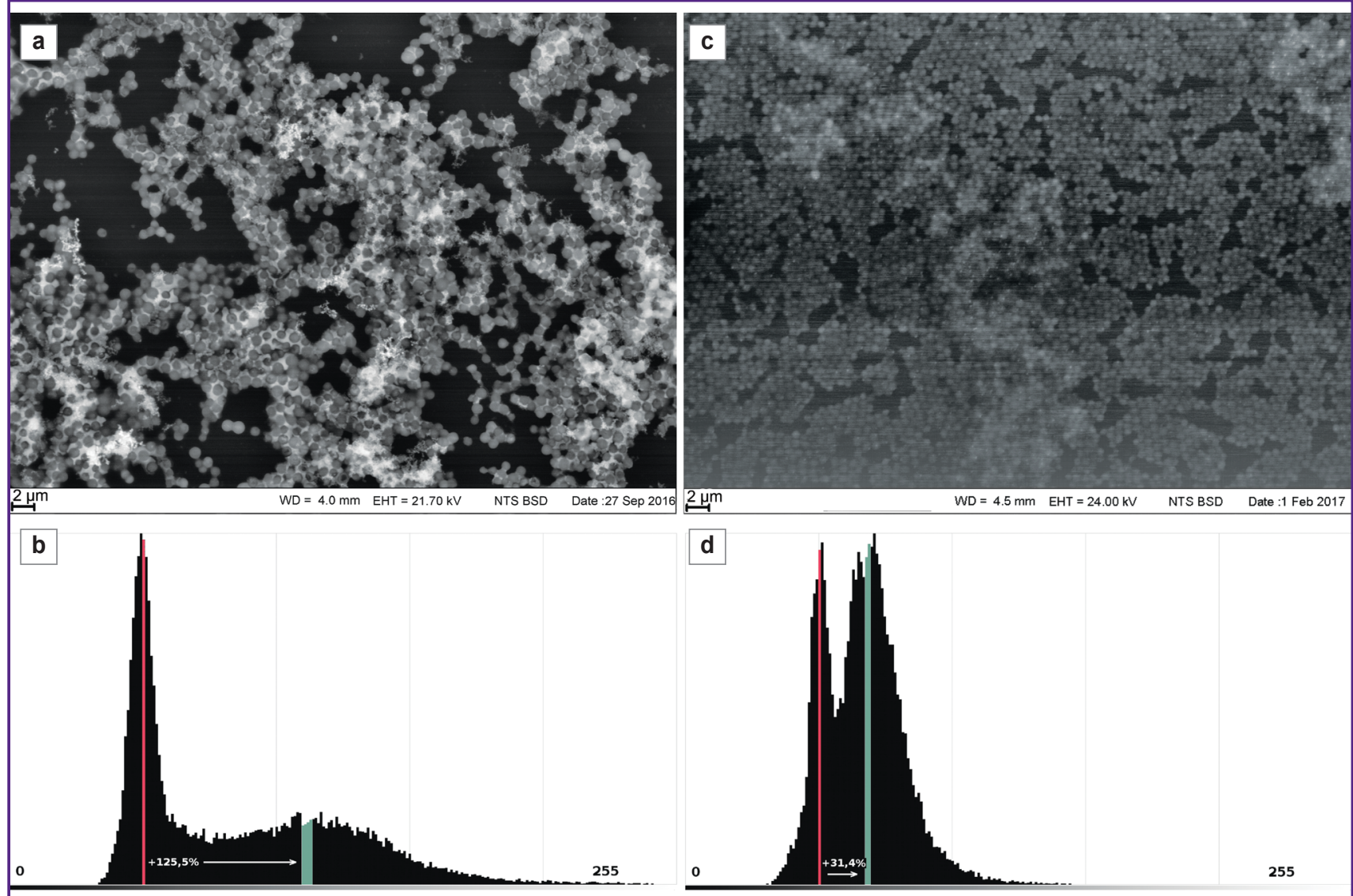

255

Figure 1. The cells of S. aureus ATCC 29213:

SEM images (a) and histograms of brightness distribution on the image (b) of specimens stained with neodymium chloride; SEM images (c) and histograms of brightness distribution on the image (d) of the native (unstained) specimens 


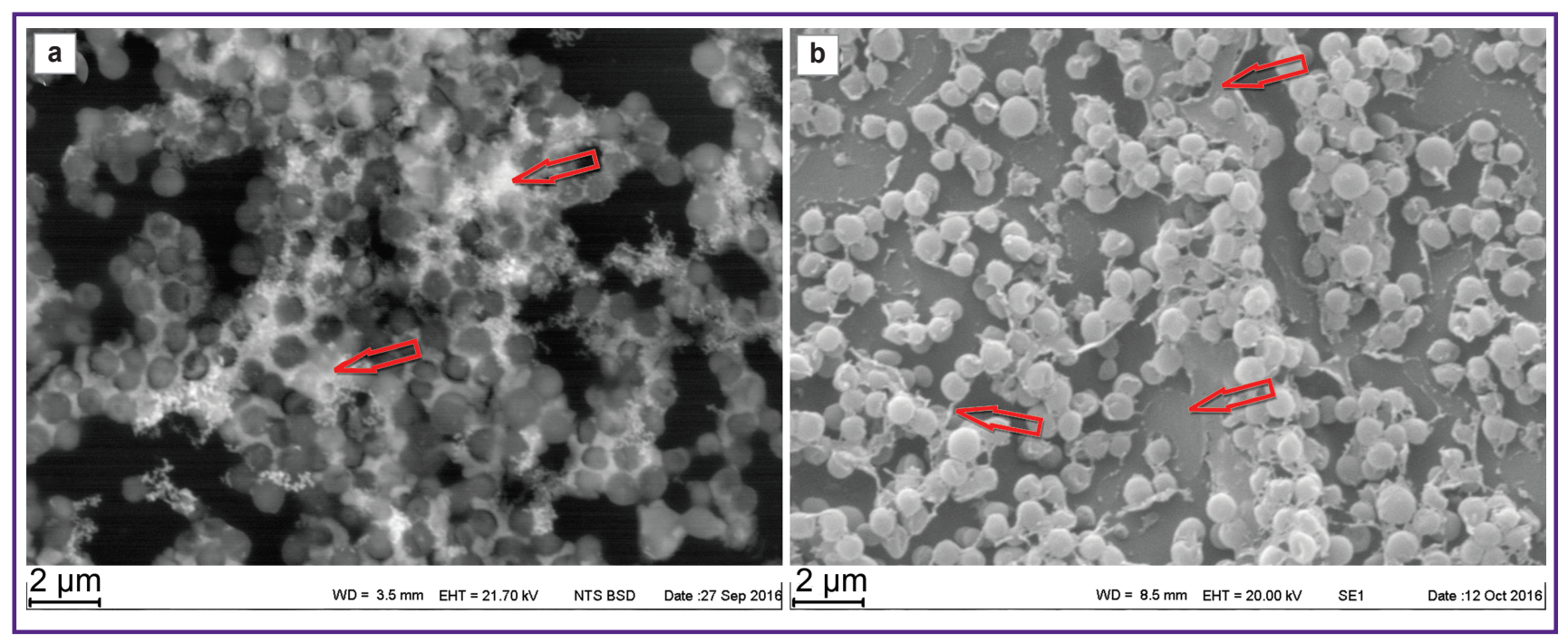

Figure 2. SEM images of S. aureus ATCC 29213 biofilms:

(a) staining with neodymium chloride; (b) traditional specimen preparation for SEM including dehydration of the specimen and metallization of its surface; extracellular biofilm matrix is shown by the arrows; see section "Materials and Methods" for the description of the microscopy procedure

the characteristic dimensions, morphology and typical cluster architecture for staphylococci. Images of staphylococci were characterized by a good contrast and a high definition of cellular borders. The extracellular (biofilm) matrix was presented by an amorphous substance, which participates in the consolidation of staphylococci into multicellular conglomerates. Of great value is the fact that such qualitative images show a relative three-dimensional position of the cells and matrix.

The traditional method of specimen preparation enabled acquisition of staphylococci biofilm images with similar characteristics of cellular elements but with a different preservation of the matrix. The matrix after the traditional preparation is not integral, has areas of discontinuity, is characterized by a loose integration with the cellular clusters.

At the third stage, the feasibility of lanthanoid staining of clinically significant microorganisms from various taxonomic groups was studied. As have already been said, S. aureus ATCC 29213 staphylococci, representatives of gram-positive bacteria, were well stained with lanthanoids (See Figure 1 (a)), were seen on the image to have a typical ball-like shape $(0.7-1.0 \mu \mathrm{m}$ in diameter), were tightly arranged into multicellular clusters. They had no capsule but were coupled with a well-contrasted intercellular substance with an amorphous structure.

Gram-positive bacteria, S. pneumoniae ATCC 49619 pneumococci (Figure $3(\mathrm{a})$ ), were characterized by a typical lancet-like shape, were arranged in the form of diplococci, and were highly contrast. Some of the cells had intracellular septa. Contrast capsule-like formations were encountered in the majority of the cells.
Representatives of gram-negative bacteria, K. pneumoniae 36-2246 enterobacteria (Figure 3 (b)), demonstrated a typical well-contrasted rod-like shape and dimensions (about $2 \mu \mathrm{m}$ long), irregular position relative each other. Some cells had low-contrast, relatively thin capsule-like formations.

Other gram-negative bacteria, glucose non-fermenting bacteria, $P$. aeruginosa ATCC 27853 (Figure 3 (c)) and A. baumannii ATCC 19606 (Figure 3 (d)), were also visualized in their typical morphological forms with irregular position of the cells relative each other. Their contours were highly contrast; some cells of $P$. aeruginosa carried a polar flagellum. Presence of capsules in $P$. aeruginosa and $A$. baumannii was not observed.

The representatives of Actinobacteria class, C. diphtheriae and biovar mitis, were located in the form of irregular aggregations, had sharp contours, rodlike shape, and the length of about $2 \mu \mathrm{m}$ (Figure $3(\mathrm{e})$ ). Brightly stained inclusions from 0.1 to $0.4 \mu \mathrm{m}$ in diameter were detected in the cytoplasm of over $80 \%$ of the cells. In some clusters, corynebacteria are coupled with each other by tiny $(0.1 \mu \mathrm{m}$ or less in diameter) filamentous structures.

Morphology of C. albicans 66-1556 fungi (Figure 3 (f)) corresponds to the yeast form of this fungus existence. Cells connected in clusters had a round form, highly contrast borders, and 3-6 $\mu \mathrm{m}$ diameter. A brightly contrasting area, the diameter of which was equal to $40 \%$ of the cell diameter, was registered in the center of more than $50 \%$ of the cells. Also brightly stained were the areas of intercellular junctions less than $0.1 \mu \mathrm{m}$ thick. 

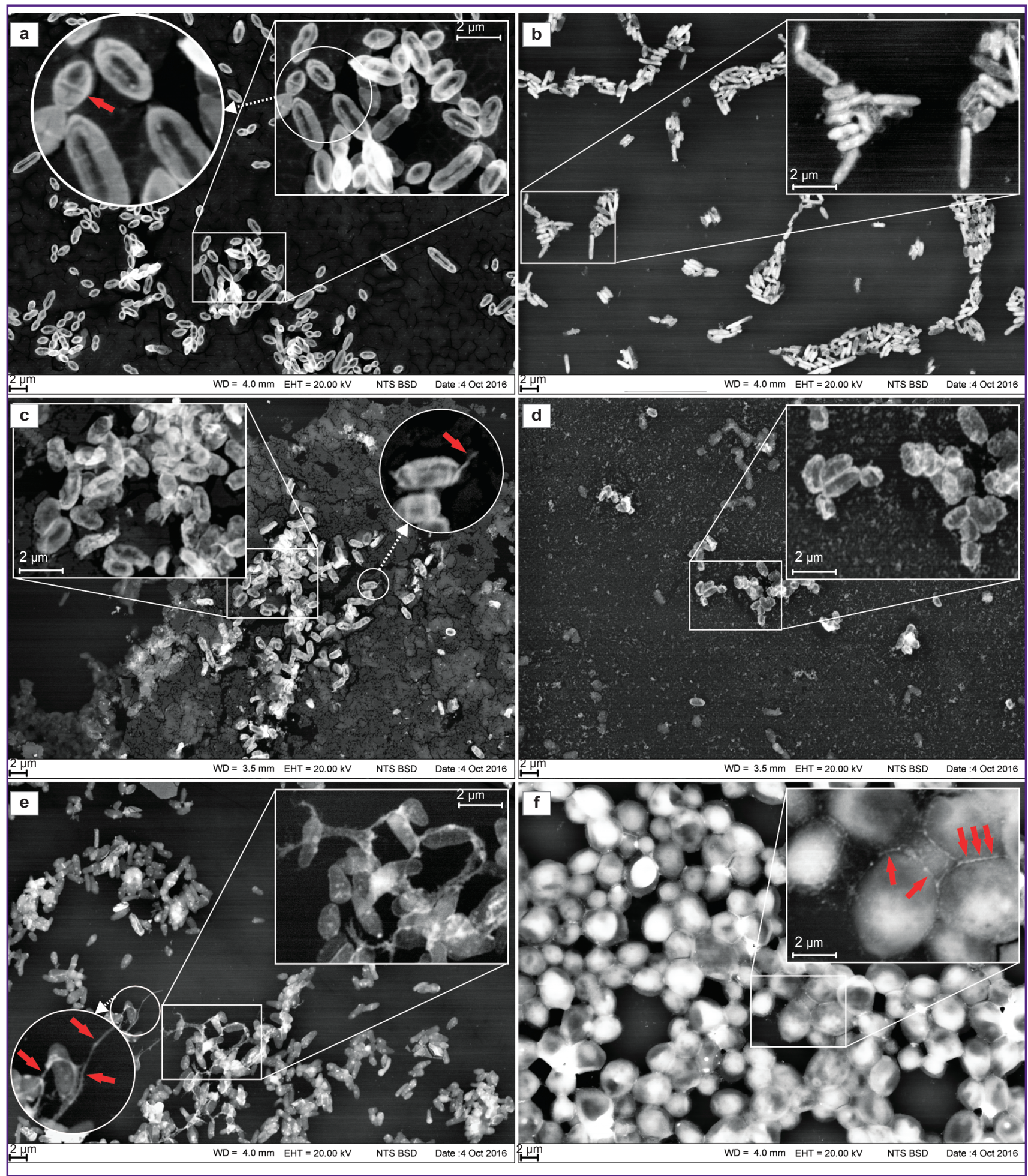

Figure. 3. Images of the representatives of various taxonomic groups of microorganisms obtained using SEM specimens stained with neodymium chloride:

(a) S. pneumoniae, the intracellular septum is shown by the arrow; (b) K. pneumoniae; (c) P. aeruginosa, the polar flagellum is indicated by the arrow; (d) A. baumannii; (e) C. diphtheriae, biovar mitis, arrows show filamentous structures with corynebacteria adhered to them (cord factor); (f) C. albicans, arrows show brightly stained intercellular junctions; see section "Materials and Methods" for the description of the microscopy procedure 
Discussion. The results obtained showed that in spite of the different character of images, visualization of microorganisms in SEM using lanthanoid staining technique is not inferior to the traditional specimen preparation with metal coatings, and the obtained images substantially surpass in the contrast the specimens without preparation. Physical, structural and biochemical aspects underlie a high image contrast. Lanthanoids, being the elements with a high atomic number, are known to provide a high density of electron backscattering under the impact of a microscope electron beam, which results in a high brightness on the scans of the biological object loci, saturated with these elements $[4,7]$. Several mechanisms of capturing and deposition of lanthanoids in biological systems are known, one of them is determined by the ability to substitute calcium in the exchange chains, the second one by the ability to actively bind to phosphate residues. In addition to phospholipid-containing membranes, lanthanoid can stain other biological structures: phosphor-containing biopolymers rich in phosphor and calcium, for example, DNA, polyphosphates (volutin), and calcium-dependent groups of proteins [8-10]. These properties allow usage of lanthanoids for visualization of live objects. The results of using lanthanoid staining of microbiological specimens obtained in this work are an experimental proof of the enumerated hypothetic premises.

The images showed that the lanthanoid stained microorganisms demonstrated morphological attributes characteristic for their taxons. In particular, the microbe membranes (cytoplasmatic membrane, the outer membrane of gram-negative bacteria) strictly reproduced the shape of the cells and reflect their real dimensions. The chromosome regions located in the center of the cells were also stained by lanthanoids owing to a great number of DNA phosphate groups. Polyphosphate volutin present in the corynebacteria was clearly visualized as well. A high structural integrity of the extracellular matrix, which contained derivatives of DNA, membranes, proteins, was observed in the specimens treated with neodymium chloride, also detected were intermicrobial junctions and position of the cells relative to each other [11]. Interesting is the fact of intracellular septa visualization, that was demonstrated on the model of pneumococci (See Figure 3 (a)). The detected septa were the sign of cell division initiation, previously they were found in bacteria only by transmission electronic microscopy on ultrathin sections [12]. Thus, application of lanthanoid staining technique in combination with a detector of back-scattered electrons makes it possible to visualize not only external structures but to peer inside the cell that was impossible using SEM with the traditional specimen preparation.

Lanthanoid staining allowed the acquisition of the original image of bacterial films. Biofilms are known to be the complex of cells and extracellular matrix [13]. It is the detection of the extracellular matrix that enables microbiologists to make a diagnosis of the biofilm process [1]. In this connection, lanthanoid staining, which reliably recognizes extracellular matrix structures (See Figure 2 (a)), may play an important role as a tool for diagnosing infections associated with biofilms.

Of special attention is a high rate of this technique: the process of treating microbes with a neodymium chloride-based agent takes $30 \mathrm{~min}$. Such a rate is one more factor promoting this method to the field of practical diagnosis.

Conclusion. The suggested method of specimen preparation for scanning electron microscopy based on staining microbial cells with lanthanoids allows visualization and assessment of the main morphological structures of microbe cells including their shape, dimensions, relative position, capsule and flagella, some intracellular structures (polyphosphate inclusions, septum during their division), as well as an extracellular matrix. This method is quickly implementable, which opens the potential of its practical application in diagnostic technologies of clinical microbiology.

Study Funding. The microbiological part of the study was supported by the Russian Foundation for Basic Research (grant 16-04-01528), electron-microscopic investigations were financed by the Federal Agency of Scientific Organizations, Russia, within the frames of R\&D works 0511-2014-0031.

Conflicts of Interest. The authors have no conflicts of interest to declare.

\section{References}

1. Chebotar I.V., Guryev E.L. Laboratory diagnostics of clinically significant microbial biofilms. Voprosy diagnostiki $v$ pediatrii 2012; 4(4): 15-20.

2. Priester J.H., Horst A.M., Van De Werfhorst L.C., Saleta J.L., Mertes L.A.K., Holden P.A. Enhanced visualization of microbial biofilms by staining and environmental scanning electron microscopy. J Microbiol Methods 2007; 68(3): 577558, https://doi.org/10.1016/j.mimet.2006.10.018.

3. Ensikat H.-J., Weigend M. Creating internal conductivity in dry biological SEM samples by a simple vapour treatment. J Microsc 2014; 256(3): 226-230, https://doi.org/10.1111/ jmi.12177.

4. Novikov I.A., Subbot A.M., Fedorov A.M., Griboedova I.G., Antonov E.N., Vakhrushev I.V. Supravital lanthanoid staining for scanning electron microscopy of biological objects. Geny i kletki 2015; 10(2): 90-96.

5. Nakakoshi M., Nishioka H., Katayama E. New versatile staining reagents for biological transmission electron microscopy that substitute for uranyl acetate. J Electron Microsc (Tokyo) 2011; 60(6): 401-407, https://doi.org/10.1093/jmicro/dfr084.

6. Pogorelov A.G., Chebotar I.V., Pogorelova V.N. Scanning electron microscopy of biofilms adherent to the inner catheter surface. Bull Exp Biol Med 2014; 157(5): 711-714, https://doi.org/10.1007/s10517-014-2648-0.

7. Novikov I.A., Subbot A.M., Kiryushchenkova N.P., Nesterova T.V., Gabashvili A.N., Sitnikov A.V., Bursov A.I. Fast and easy method of lanthanoid staining for visualization of cellular ultrastructure and spatial arrangement. AIP Conference Proceedings 2016; 1748(1): 020009, https://doi. org/10.1063/1.4954343. 
8. Doggenweiler C.F., Frenk S. Staining properties of lanthanum on cell membranes. Proc Natl Acad Sci USA 1965; 53(2): 425-430, https://doi.org/10.1073/pnas.53. 2.425 .

9. Shaklai M., Tavassoli M. Lanthanum as an electron microscopic stain. J Histochem Cytochem 1982; 30(12): 13251330, https://doi.org/10.1177/30.12.6185564.

10. Evans C.H. Biochemistry of the lanthanides. New York: Springer Science \& Business Media; 2013; 453 p.

11. Chebotar I.V., Mayansky A.N., Mayansky N.A.
Matrix of microbial biofilms. Klinicheskaya mikrobiologiya $i$ antimikrobnaya khimioterapiya 2016; 18(1): 9-19.

12. Rybal'chenko O.V., Bondarenko V.M., Dobritsa V.P. Atlas ul'trastruktury mikrobioty kishechnika cheloveka [Atlas of the ultrastructure of human gut microbiota]. Saint Petersburg: IITs VMA; 2008; 112 p.

13. Donlan R.M., Costerton J.W. Biofilms: survival mechanisms of clinically relevant microorganisms. Clin Microbiol Rev 2002; 15(2): 167-193, https://doi.org/10.1128/ cmr.15.2.167-193.2002. 